\title{
Antimicrobial Susceptibility Trends in Salmonella enterica Isolates - A 6 Year Study from a Tertiary Care Hospital in North India
}

\author{
Eshani Dewan¹, Vandana Verma² \\ ${ }^{1}$ Assistant Professor, Department of Microbiology, Christian Medical College Hospital, Ludhiana, Punjab, India. \\ ${ }^{2}$ Professor and HOD, Department of Microbiology, Christian Medical College Hospital, Ludhiana, Punjab, India.
}

\section{ABSTRACT}

\section{BACKGROUND}

Enteric fever is a major cause of morbidity and mortality in tropical areas worldwide. The Indian subcontinent bears the major burden of the disease, both in terms of absolute case numbers and drug-resistant strains. In this study, we aimed to investigate the incidence of enteric fever associated with Salmonella enterica and determine its antimicrobial susceptibilities to therapeutic antimicrobials in a tertiary care teaching hospital of Punjab.

\section{METHODS}

This retrospective and prospective study was conducted at the Department of Microbiology, CMC and Hospital, Ludhiana. All the culture-positive enteric fever cases from June 2013 to June 2019 presenting to our hospital were included in the study. Antimicrobial susceptibility was done as per corresponding CLSI guidelines.

\section{RESULTS}

A total of 944 strains of Salmonella species- Salmonella Typhi 772 (81.78\%) and Salmonella Paratyphi A 172(18.22\%) were isolated from the blood cultures. Antimicrobial susceptibility for chloramphenicol, ampicillin and co-trimoxazole was found to be $91.97 \%, 98.58 \%$ and $100 \%$ respectively for $S$. Typhi strains, whereas it was $94.19 \%, 97.09 \%$ and $100 \%$, respectively for $S$. Paratyphi $A$ isolates. Ciprofloxacin and ofloxacin susceptibility were $92.23 \%$ and $97.67 \%$ for S. Typhi, $86.63 \%$ and $98.84 \%$ for $S$. Paratyphi $A$ respectively. There is a continuous increase in ciprofloxacin minimum inhibitory concentration values over the time. Majority (98.51\%) of Salmonella isolates were nalidixic acid resistant. Although the rate of multi-drug resistant (MDR) Salmonella strains was nil but their reduced susceptibility to fluoroquinolones has restricted their routine empirical use.

\section{CONCLUSIONS}

Enteric fever continues to cause significant morbidity due to delayed diagnosis, inadequate treatment and worsening drug resistance in India and beyond. There has been a reported decline in MDR with a parallel increase in decreased ciprofloxacin susceptibility among $S$. Typhi. Third generation cephalosporins are the safest choice for empirical use as ampicillin, chloramphenicol or cotrimoxazole are less likely to be preferred because of longer duration of therapy, threat of reemergence of resistance, side effects and higher relapse rates. Judicious use of these antibiotics is mandatory to prevent emergence of resistant strains.

\section{KEY WORDS}

Enteric Fever, Salmonella Typhi, Salmonella Paratyphi A, MDRT, DCS
Corresponding Author: Dr. Eshani Dewan, Department of Microbiology, Christian Medical College Hospital, Ludhiana, Punjab, India.

E-mail: eshani.dewan@gmail.com

DOI: $10.14260 /$ jemds/2019/677

Financial or Other Competing Interests: None.

How to Cite This Article:

Dewan E, Verma V. Antimicrobial susceptibility trends in salmonella enterica isolates- a 6 year study from a tertiary care hospital in North India. Evolution Med. Dent. Sci. 2019;8(42):3119-3124, DOI: 10.14260/jemds/2019/677

Submission 19-08-2019, Peer Review 29-09-2019,

Acceptance 05-10-2019,

Published 21-10-2019. 


\section{BACKGROUND}

Enteric fever is a systemic infection classically caused by Salmonella enterica subspecies enteric serovar typhi $(S$. Typhi) and a very similar but often less severe disease caused by Salmonella enterica subspecies enterica serovar Paratyphi A (S. Paratyphi A).[1] S. Paratyphi B predominates in Europe; $S$. Paratyphi $C$ is rare and is seen only in the Far East.[2] These organisms are important causes of acute, potentially lifethreatening febrile illness among crowded and impoverished populations with poor sanitation who are exposed to unsafe water and food and impose a risk to travellers visiting endemic areas. ${ }^{[3]} S$. Typhi, is a highly adapted human specific pathogen that has evolved over decades and has remarkable mechanisms for persistence in its host.[4] Salmonella is transmitted by faeco-oral ingestion. In most cases, salmonellosis is caused by consumption of contaminated food products, especially those of animal origin. Fruits and vegetables also have been reported as vehicles in Salmonella transmission and contamination can occur at different steps along the food chain.[5] The ensuing disease is a non-specific febrile illness which affects an estimated 12 to 27 million people worldwide per year, resulting in approximately $1 \%$ deaths annually.[6,7,8] Although over the last century a dramatic reduction in incidence has been achieved in most high-income countries, but the continued inadequate access to clean and hygienic water and rapid intercontinental spread of antibiotic-resistant strains hampers disease control efforts, especially in the third world countries.[8,9] The current burden of disease is highest among children and young adults in South and Southeast Asia.[6,7,8] A vast disparity is seen in the global incidence rates from $<0.1$ per 100,000 person years in North America to 976 per 100,000 person years in South Asia.[7] The Indian sub-continent bears the major burden of disease with an estimated 6,345,776 cases per year and remains the typhoid capital of the world.[6]

The incidence also shows seasonal variation with peaks occurring between May and October, overlapping with the monsoon season, closely reflecting the pattern seen with other water borne diseases. Baseline endemicity is punctuated by intermittent epidemics that may occur through the year. [10,11]

The major challenge in enteric fever at present is the increase in antimicrobial resistance in $S$. Typhi and $\mathrm{S}$. Paratyphi $A$, especially that towards fluoroquinolones. The reports on ciprofloxacin resistance started to appear soon after the rise of multi-drug resistant Salmonella Typhi (MDRT).It was observed that fluoroquinolones had good in vitro and in vivo activity against salmonellae and hence rapidly became the drugs of choice in cases of MDR salmonellosis. ${ }^{[4]}$ This was soon followed by the appearance of isolates with low-level resistance (MIC $\geq 0.25 \mu \mathrm{g} / \mathrm{mL}$, but $<4 \mu \mathrm{g} / \mathrm{mL}$ ) to fluoroquinolones.[12,13]The regular revisions of Clinical and Laboratory Standards Institute (CLSI) guidelines in the interpretative criteria of 2011 and the addition of new fluoroquinolones in 2015 and 2016 indicate the urgency and need to revise breakpoints to optimize the dose of fluoroquinolones and to use this drug effectively in susceptible isolates. [14] The treatment is getting more difficult due to the compounded fact that even though at present, ceftriaxone is the best available drug, it has also started to show an increasing trend of minimum inhibitory concentration (MIC) values against Salmonella enterica serovar Typhi and S. enterica serovar Paratyphi A.[15,16,17] Most clinicians now prefer azithromycin for uncomplicated disease and ceftriaxone for patients requiring intravenous therapy and this is also recommended in the National Treatment Guidelines for Antimicrobial Use in Infectious Diseases.[18] Over the last decade there have been a few sporadic reports of typhoid resistant to third generation cephalosporins but these isolates are uncommon.[19] Another interesting observation made at many Indian centers has been a reversal in resistance trends with rates of MDRT falling rapidly with increased isolation of decreased ciprofloxacin susceptibility (DCS) isolates.[20]

This study was conducted to determine the spectrum of Salmonella enterica serovars isolated from the blood culture of the patients suffering from enteric fever and their antibiotic susceptibility pattern to commonly used antibiotics in a community based tertiary care teaching hospital in Punjab.

\section{METHODS}

We carried out a laboratory-based retrospective and prospective analysis over a period of 6 years from $27^{\text {th }}$ June 2013 to $26^{\text {th }}$ June 2019 in the Department of Microbiology at Christian Medical College \& Hospital, Ludhiana. A total sample of 53,398 blood cultures processed during this period by BACTEC 9120 and 9050 automated culture system; Becton Dickinson were included in the study. The diagnosis of enteric fever was made by using standard blood culture protocols and biochemical methods for identification were followed. Isolates were confirmed using specific Salmonella antisera (Denka Seiken Co., Ltd. Tokyo, Japan).

Antibiotic susceptibility testing was performed by modified Kirby-Bauer disc diffusion test and minimum inhibitory concentrations (MICs) were determined using the the MicroScan WalkAway 96 system, referring to the Clinical and Laboratory Standards Institute guidelines for the corresponding year of isolation. [21-27]The antibiotic discs used were Ampicillin $(10 \mu \mathrm{g})$, chloramphenicol $(30 \mu \mathrm{g})$, cotrimoxazole $(1.25 / 23.75 \mu \mathrm{g})$, ciprofloxacin $(5 \mu \mathrm{g})$, ofloxacin (5 $\mu \mathrm{g})$, nalidixic acid $(30 \mu \mathrm{g})$, amikacin $(10 \mu \mathrm{g})$, gentamicin $(10 \mu \mathrm{g})$, cefotaxime (30 $\mu \mathrm{g})$,ceftriaxone (30 $\mu \mathrm{g})$, cefoperazone (75 $\mu \mathrm{g})$, ceftazidime $(30 \mu \mathrm{g})$, piptaz $(100 / 10 \mu \mathrm{g})$, cefoperazone/sal $(30 / 10 \mu \mathrm{g})$, azithromycin $(15 \mu \mathrm{g})$ (Hi-media Laboratories Ltd, Mumbai, India). MIC for ofloxacin was determined in the strains after the recommendation of these antimicrobial agents for enteric fever in 2013.[22] MIC for azithromycin was determined in the strains isolated after 2015 when the breakpoints were defined by CLSI.[24] All Salmonella isolates were also subjected to double disk test for detection of extended spectrum beta-lactamases (ESBL). Escherichia coli ATCC 25922 and Staphylococcus aureus ATCC 25923 were used for the quality control of antimicrobial susceptibility testing.

\section{Statistical Analysis}

Descriptive statistics was used to summarize demographic and other clinical features of patients. Qualitative and 
quantitative data values were expressed as frequency along with percentage.

\section{RESULTS}

In our study, several interesting trends were observed. During the 6 years study period, a total of 53,398 blood cultures were received. Of these, $944(1.76 \%)$ were culture positive for typhoidal salmonellae. The predominant serotype obtained was S. Typhi $(772,81.78 \%)$ followed by S. Paratyphi $A(172,18.22 \%)$ shown in Figure 1. Figure 2 shows the year wise distribution of Salmonella isolates. The male-to-female ratio for the culture-positive cases was 2.41:1 (667 male and 277 female) Figure 3. The age-wise distribution showed that $98(10.38 \%)$ cases occurred in $<5$ years age group, 602 $(63.77 \%)$ cases in $5-18$ years age group and $244(25.85 \%)$ were found in $>18$ years of age, shown in Figure 4 . Typhoid fever cases occurred in all months throughout the year, however they peaked during the months of May-September each year.
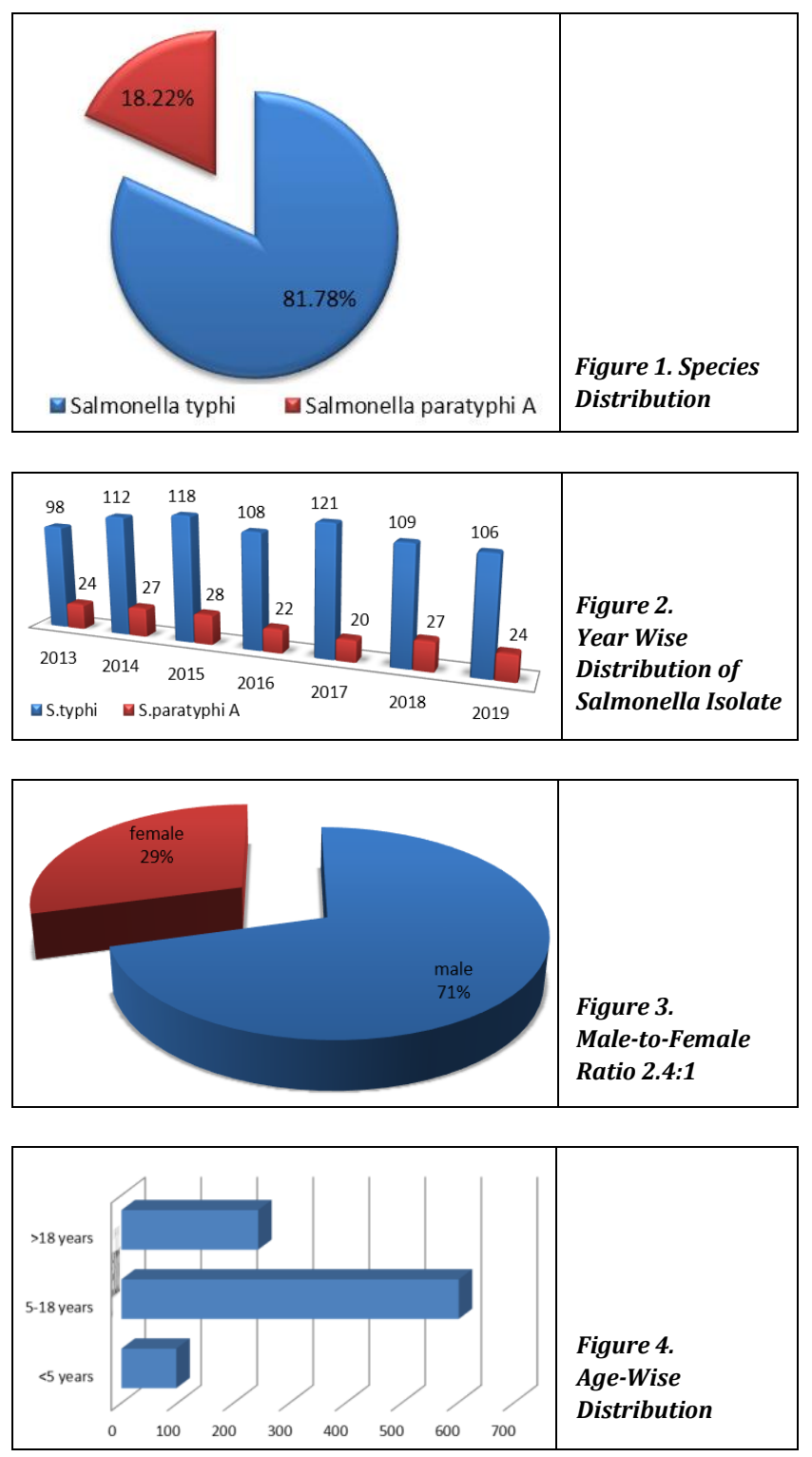

S. Typhi and S. Paratyphi $A$ were both found to be $100 \%$ susceptible to co-trimoxazole, amikacin and gentamicin. $S$. Typhi showed higher susceptibility to azithromycin (98.96\%) and ampicillin (98.58\%), whereas $S$. Paratyphi $A$ strains had $96.511 \%$ and $97.09 \%$ sensitivity respectively. Chloramphenicol susceptibility was the lowest among all antibiotics among S. Typhi isolates (91.97\%). Resistance to nalidixic acid (NA) was found to be high; it has been rising each year with an average resistance of $98.51 \%$ and was noticed to be a staggering $100 \%$ in 2018 \& 2019. Interestingly a continuous ciprofloxacin increase in minimum inhibitory concentration (MIC) values was noticed over the period of time (MICs $0.125-0.5 \mu \mathrm{g} / \mathrm{mL}$ ). A positive correlation was observed between reduced ciprofloxacin susceptibility and nalidixic acid resistance in all the isolates. S. Paratyphi $A$ strains showed higher rate (99.42\%) of NA resistance than $S$. Typhi (98.31\%). The antibiotic susceptibility is shown in Table 1.

\begin{tabular}{|c|c|c|c|c|c|c|}
\hline Antibiotics & \multicolumn{3}{|c|}{ S. Typhi } & \multicolumn{3}{c|}{ S. Paratyphi A } \\
\hline & S & I & R & S & I & R \\
\hline Co-trimoxazole & 772 & 0 & 0 & 172 & 0 & 0 \\
\hline Amikacin & 772 & 0 & 0 & 172 & 0 & 0 \\
\hline Gentamicin & 772 & 0 & 0 & 172 & 0 & 0 \\
\hline Ceftazidime & 772 & 0 & 0 & 170 & 1 & 1 \\
\hline Cefotaxime & 770 & 0 & 2 & 171 & 1 & 0 \\
\hline Cefoperazone & 769 & 0 & 3 & 172 & 0 & 0 \\
\hline Piperacillin-Tazobactam & 768 & 1 & 3 & 168 & 3 & 1 \\
\hline Cefoperazone Sulbactam & 768 & 1 & 3 & 171 & 1 & 0 \\
\hline Ceftriaxone & 768 & 2 & 2 & 171 & 0 & 1 \\
\hline Azithromycin & 764 & 4 & 4 & 166 & 2 & 4 \\
\hline Ampicillin & 761 & 2 & 9 & 167 & 1 & 4 \\
\hline Ofloxacin & 754 & 0 & 18 & 170 & 0 & 2 \\
\hline Ciprofloxacin & 712 & 39 & 21 & 149 & 19 & 4 \\
\hline Chloramphenicol & 710 & 60 & 2 & 162 & 10 & 0 \\
\hline Nalidixic acid & 13 & 0 & 759 & 1 & 0 & 171 \\
\hline Table
\end{tabular}

Table 1. Antimicrobial Susceptibilities of Salmonella Enterica Serovars $S$ : sensitive, $I$ : intermediate sensitive, $R$ : resistant.

Overall, $92.23 \%$ and $86.63 \%$ of Salmonella Typhi and para Typhi serovars were susceptible to ciprofloxacin, while $97.67 \%$ and $98.84 \%$ of them were susceptible to ofloxacin. Reduced susceptibility to ciprofloxacin $(2.72 \%$ and $2.33 \%$ resistant, $5.05 \%$ and $11.05 \%$ intermediate) was observed in both S. Typhi and S. Paratyphi $A$ isolates respectively. Ofloxacin also showed a similar pattern with $2.33 \%$ and $1.16 \%$ resistance toS. Typhi and S. Paratyphi A, respectively, although none of the strains showed intermediate susceptibility to the drug.

Salmonella Typhi isolates were highly susceptible to ceftazidime (100\%), cefotaxime (99.74\%), cefoperazone (99.61\%) and 99.48\% to piptaz, cefoperazone sulbactam and ceftriaxone. Whereas Salmonella Paratyphi $A$ isolated were $100 \%$ susceptible to cefoperazone, followed by cefoperazone sulbactam, cefotaxime and ceftriaxone (99.42\%), ceftazidime (98.84\%) and piperacillin-tazobactam (97.67\%). A continuous increase in ceftriaxone minimum inhibitory concentration (MIC) 50 and MIC $_{90}$ values was noticed over the time. MIC pattern was observed for ceftriaxone from 0.032 to 0.94 in S. Typhi followed by $0.019-0.75$ over the years. MIC to ceftriaxone in typhoidal salmonellae is gradually moving towards resistance and more data are required to fully understand the changing susceptibility pattern.

No ESBL or multidrug-resistant strains were detected in any of isolates. 


\section{DISCUSSION}

Enteric fever remains a major cause of febrile illness among the urban population of endemic countries with limited water and sanitation infrastructure.[28] World Health Organization (WHO) has recommended vaccination with the existing $\mathrm{Vi}$ polysaccharide vaccine targeting high-risk areas of typhoid fever as a preventive measure.[29] Besides, estimation of the disease burden and its aetiology along with antimicrobial susceptibilities would be helpful in the development of effective prevention and control interventions. India is a vast country with considerable geographic and social diversity. However, enteric fever is endemic throughout the nation and places a heavy burden on both the government and private healthcare sector. The incidence varies both geographically from 140 episodes per 100,000 person years in Kolkata[30] to 270 per 100,000 person years in the capital.[31] The International Vaccine Institute conducted a study where the incidence of culture-proven S. Typhi was found to be 340 per 100,000 population-year among children between 2 to 5 years, 493/100,000 population-year in children aged 5-15 years and 120/100,000 population-year in adults $>15$ years. [32] In our study the males in the age group of 5-18 years were the most affected. This data is well supported by other studies in India.[33] Usually children 15 years of age and younger are more susceptible, most probably because adults develop immunity from recurrent infection and sub-clinical cases.

Overall, the incidence rate of enteric fever caused by serovars of Salmonella enterica in our hospital was $1.76 \%$. Similar incidence was reported by Prajapati et al. (2.55\%); on the other hand higher rates were reported by Maheshwari et al. (9.81\%).[34,35] The lower rates of blood-culture-positive enteric fever might be due to the use of antibiotics prior to the blood culture and low blood volume used for culture $(10$ $\mathrm{ml}$ for adult and $5 \mathrm{ml}$ for children). However, we did not evaluate the prior antibiotic consumption by the patients before enrolment.

S. Typhi outnumbered S. Paratyphi A with approximately 5 times higher rate of isolation as also found in studies from other parts of India.[10,36] Males were more infected than females with M: F ratio of 2.41:1. This could be due to our cultural background where men are more likely to report to the hospital; at the same time they are more likely to acquire infection due to more outdoor activities. This correlates with the studies of Singhal et al. and Prajapati et al.[10,34] Although the disease occurred throughout the year, there was an increase from May to September months. This is in concordance with other reports from India which have related increased transmission to rainfall and water contamination. [10,11]

Antibiotic resistance has raised its ugly head whenever a drug has been widely used for the treatment of enteric fever, in India. Chloramphenicol resistance was seen worldwide within 2 years of its introduction in 1948, although the first outbreak in India was not reported much later in 1972.[37] Use of co-trimoxazole promoted resistance through sul1 and sul2 and the dfra7 gene. MDRT was defined as isolates resistant to amoxicillin, co-trimoxazole and chloramphenicol towards the end of 1980s and rampant throughout India. Singhal et al. reported, MDRT initially in the earlier part of 1990 but by the last quarter of the same year, $100 \%$ of $S$.
Typhi isolates were multidrug-resistant. [10] The high rates of MDR strains resulted in the increased use of fluoroquinolones (Ofloxacin and ciprofloxacin), also they are available for oral use and are less expensive options. [38] However, they are increasingly becoming ineffective in enteric fever cases due to the emergence of nalidixic acid resistant (NAR) strains. [39] In our study, rate of NAR, a phenotypic marker for reduced susceptibility to fluoroquinolones, was very high $(98.52 \%)$. Moreover, S. Paratyphi strains showed even higher rate (99.42\%) of NAR than S. Typhi (98.32\%). Similar rates of resistance to nalidixic acid (NA) resistant isolates were reported in the studies through the Indian subcontinent by Singhal et al, Menezes et al and Veeraraghavan et al.[10,39,40]

A rise in DCS accompanied with a sustained decrease in the MDR typhoidal Salmonella has been noticed recently.[14] We have also had similar observations in our study. A study from North India reported the declining MDR rates with the increased incidence of nalidixic acid resistant among DCS isolates. [10] There has been a reported reduction in MDR rated with a parallel rise in DCS among S. Typhi. Ampicillin, chloramphenicol or cotrimoxazole are less likely preferred due to longer duration of therapy, threat of re-emergence of resistance, higher relapse rates and more side effects.

Besides fluoroquinolones, the overall susceptibility of Salmonella isolates to chloramphenicol was found to be lowest (91.97\% for S. Typhi and $94.19 \%$ for S. Paratyphi A). Susceptibility of Salmonella isolates to other first-line drugs, that is, ampicillin, cotrimoxazole, and azithromycin, was also excellent, $98.31 \%, 100 \%$, and $98.52 \%$, respectively. The decreased use of first-line antibiotics in treating salmonellosis and other infections could be the most probable reason for this re-emergence of susceptibility.[41]

We observed that cephalosporins (Ceftriaxone, cefotaxime, and cefixime) exhibited excellent efficacy towards isolated Salmonella serovars with more than 99\% sensitivity. To avoid clinical failures, third generation cephalosporins are now preferred for the treatment of MDR and nalidixic acid-resistant isolates due the rising DCS phenomenon. Although very low at present $(1 \%)$, there is a gradual emergence of resistance among cephalosporins that being observed sporadically with their increased use [42,43] This emphasises the importance of this group of antibiotic as a reserve drug for treating MDR and ciprofloxacin resistant cases. Fluoroquinolones would still be the effective therapeutic regimen in our scenario because a good proportion of quinolones is found susceptible, but susceptibility test should be performed before starting the quinolone therapy. Clinicians have to take utmost precaution as increased use of cephalosporin or azithromycin in treating fluoroquinolone resistant $S$. Typhi may give rise to cephalosporin resistance or azithromycin treatment failure. In the era of MDR, combination therapy could be the best alternative for successfully treating enteric fever cases.

\section{CONCLUSIONS}

Enteric fever continues to cause significant morbidity due to delayed diagnosis, inadequate treatment and worsening drug resistance in India and beyond. There has been a reported decline in MDR with a parallel increase in decreased ciprofloxacin susceptibility among S. Typhi. Third generation 
cephalosporins are the safest choice for empirical use as ampicillin, chloramphenicol or cotrimoxazole are less likely to be preferred because of longer duration of therapy, threat of re-emergence of resistance, side effects and higher relapse rates. Judicious use of these antibiotics is mandatory to prevent emergence of resistant strains.

\section{REFERENCES}

[1] John J, Van Aart CJ, Grassly NC. The burden of typhoid and paratyphoid in India: systematic review and metaanalysis. PLoS Negl Trop Dis 2016;10(4):e0004616.

[2] Bhutta ZA. Current concepts in the diagnosis and treatment of typhoid fever. BMJ 2006;333(7558):78-82.

[3] Whitaker JA, Franco-Paredes C, Del Rio C, et al. Rethinking typhoid fever vaccines: Implications for travellers and people living in highly endemic areas. J Travel Med 2009;16(1):46-52.

[4] Pegues DA, Ohl ME, Miller SI. Salmonella species, including Salmonelltyphi. In: Mandell GL, Bennett JE, Dolin R, eds. Principles and practice of infectious diseases. $6^{\text {th }}$ edn. New York: Churchill Livingstone 2005: p. 2636-54.

[5] Gomez TM, Motarjemi Y, Miyagawa S, et al. Foodborne salmonellosis. World Health Stat Q 1997;50(1-2):81-9.

[6] Crump JA, Mintz ED. Global trends in typhoid and paratyphoid fever. Clin Infect Dis 2010;50(2):241-6.

[7] Buckle GC, Walker CL, Black RE. Typhoid fever and paratyphoid fever: systematic review to estimate global morbidity and mortality for 2010. J Glob Health 2012;2(1):010401.

[8] Mogasale V, Maskery B, Ochiai RL, et al. Burden of typhoid fever in low-income and middle-income countries: a systematic, literature based update with risk-factor adjustment. Lancet Glob Health 2014;2(10):e570-e80.

[9] Arndt MB, Mosites EM, Tian M, et al. Estimating the burden of paratyphoid a in Asia and Africa. PLoS Negl Trop Dis 2014;8(6):e2925.

[10] Singhal L, Gupta PK, Kale P, et al. Trends in antimicrobial susceptibility of Salmonella Typhi from North India (2001-2012). Indian J Med Microbiol 2014;32(2):149-52.

[11] Gautam V, Gupta NK, Chaudhary U, et al. Sensitivity pattern of Salmonella serotypes in Northern India. Braz J Infect Dis 2002;6(6):281-7.

[12] Mehta G, Randhawa VS, Mohapatra NP. Intermediate susceptibility to ciprofloxacin in Salmonella Typhi strains in India. Eur J Clin Microbiol Infect Dis 2001;20(10):7601.

[13] Crump JA, Barrett TJ, Nelson JT, et al. Re-evaluating fluoroquinolone breakpoints for Salmonella enterica serotype Typhi and for non-typhi salmonellae. Clin Infect Dis 2003;37(1):75-81.

[14] Balaji V, Sharma A, Ranjan P, et al. Revised ciprofloxacin breakpoints for Salmonella Typhi: Its implications in India. Indian J Med Microbiol 2014;32(2):161-3.

[15] Renuka K, Kapil A, Kabra SK, et al. Reduced susceptibility to ciprofloxacin and Gyra gene mutation in North Indian strains of Salmonella enterica serotype Typhi and serotype Paratyphi A. Microb Drug Resist 2004;10(2):146-53.
[16] Sharma P, Dahiya S, Balaji V, et al. Typhoidal salmonellae: use of multi-locus sequence typing to determine population structure. PLoS One 2016;11(9):e0162530.

[17] Dahiya S, Sharma P, Kumari B, et al. Characterisation of antimicrobial resistance in Salmonellae during 20142015 from four centres across India: an ICMR antimicrobial resistance surveillance network report. Indian J Med Microbiol 2017;35(1):61-8.

[18] National Centre for Disease Control. Directorate General of Health Services, Ministry of Health \& Family Welfare, Government of India. National Treatment Guidelines for Antimicrobial Use in Infectious Diseases - Version 1.0 (2016). 2016.

[19] Uma B, Prabhakar K, Rajendran S, et al. Prevalence of extended spectrum beta lactamases in Salmonella species isolated from patients with acute gastroenteritis. Indian J Gastroenterol 2010;29(5):201-4.

[20] Badiyal A, Kumar Y, Sharma A, et al. Re-emergence of chloramphenicol sensitive isolates of Salmonella enterica serovar Typhi isolates in India during 2013-14. International Journal of Current Microbiology and Applied Sciences 2015;4(6):1081-6.

[21] Clinical and Laboratory Standards Institute (CLSI). Performance standards for antimicrobial susceptibility testing; approved standard; 22nd informational supplement. CLSI document M100-S22. Clinical and Laboratory Standards Institute, Wayne, PA, 2012.

[22] Clinical and Laboratory Standards Institute (CLSI). Performance standards for antimicrobial susceptibility testing; approved standard; 23rd informational supplement. CLSI document M100-S23. Clinical and Laboratory Standards Institute, Wayne, PA, 2013.

[23] Clinical and Laboratory Standards Institute (CLSI). Performance standards for antimicrobial susceptibility testing; approved standard; 24th informational supplement. CLSI document M100-S24. Clinical and Laboratory Standards Institute, Wayne, PA, 2014.

[24] Clinical and Laboratory Standards Institute (CLSI). Performance standards for antimicrobial susceptibility testing; approved standard; 25th informational supplement. CLSI document M100-S25. Clinical and Laboratory Standards Institute, Wayne, PA, 2015.

[25] Clinical and Laboratory Standards Institute (CLSI). Performance standards for antimicrobial susceptibility testing; approved standard; 26th informational supplement. CLSI document M100-S26. Clinical and Laboratory Standards Institute, Wayne, PA, 2016.

[26] Clinical and Laboratory Standards Institute (CLSI). Performance standards for antimicrobial susceptibility testing; approved standard; 27th informational supplement. CLSI document M100-S27. Clinical and Laboratory Standards Institute, Wayne, PA, 2017.

[27] Clinical and Laboratory Standards Institute (CLSI). Performance standards for antimicrobial susceptibility testing; approved standard; 28th informational supplement. CLSI document M100-S28. Clinical and Laboratory Standards Institute, Wayne, PA, 2018.

[28] Mogasale V, Mogasale VV, Ramani E, et al. Revisiting typhoid fever surveillance in low and middle income countries: lessons from systematic literature review of 
population-based longitudinal studies. BMC Infectious Diseases 2016;16:35.

[29] WHO: Background document: the diagnosis, treatment and prevention of typhoid fever. In, vol. WHO/V\&B/03.07. Geneva: World Health Organization, 2003.

[30] Sur D, Ali M, von Seidlein L, et al. Comparisons of predictors for typhoid and paratyphoid fever in Kolkata, India. BMC Public Health 2007;7:289.

[31] Sinha A, Sazawal S, Kumar R, et al. Typhoid fever in children aged less than 5 years. Lancet 1999;354(9180):734-7.

[32] Ochiai RL, Acosta CJ, Danovaro-Holliday MC, et al. A study of typhoid fever in five Asian countries: disease burden and implications for controls. Bull World Health Organ 2008;86(4):260-8.

[33] Dhadwal BS, Shetty RA. Epidemiological investigation of a typhoid outbreak. Med J Armed Forces India 2008;64(3):241-2.

[34] Prajapati K, Gamit M, Vegad M. Antimicrobial susceptibility pattern of salmonella isolates at tertiary care hospital, Ahmedabad. Int J Curr Microbiol App Sci 2018;7(06):156-60.

[35] Maheshwari V, Kaore NM, Ramnani VK, et al. A comparative evaluation of different diagnostic modalities in the diagnosis of typhoid fever using a composite reference standard: a tertiary hospital based study in central India. J Clin Diagn Res 2016;10(10):DC01-DC04.

[36] Sharma P, Dahiya S, Manral N, et al. Changing trends of culture-positive typhoid fever and antimicrobial susceptibility in a tertiary care North Indian Hospital over the last decade. Indian J Med Microbiol 2018;36(1):70-6.

[37] Paniker CK, Vimala KN. Transferable chloramphenicol resistance in Salmonella typhi. Nature 1972;239(5367):109-10.

[38] Humphries RM, Fang FC, Aarestrup FM, et al. In vitro susceptibility testing of fluoroquinolone activity against salmonella: recent changes to CLSI standards. Clinical Infectious Diseases 2012;55(8):1107-13.

[39] Menezes GA, Harish BN, Khan MA, et al. Antimicrobial resistance trends in blood culture positive Salmonella Typhi isolates from Pondicherry, India, 2005-2009. Clinical Microbiology and Infection 2012;18(3):239-45.

[40] Veeraraghavan B, Pragasam AK, Bakthavatchalam YD, et al. Typhoid fever: issues in laboratory detection, treatment options \& concerns in management in developing countries. Future Science OA 2018;4(6):FSO312.

[41] Shrestha KL, Pant ND, Bhandari R, et al. Re-emergence of the susceptibility of the Salmonella spp. isolated from blood samples to conventional first line antibiotics. Antimicrobial Resistance and Infection Control 2016;5:22.

[42] Kumar NDR, Prabaa DMS, Abirami BS, et al. Draft genome sequence of blaTEM-1 mediated cephalosporin resistant Salmonella Typhi from blood stream infection. J Glob Antimicrob Resist 2016;7:11-12.

[43] Rodrigues C, Kapil A, Sharma A, et al. Whole-genome shotgun sequencing of cephalosporin resistant Salmonella enterica Serovar Typhi. Genome Announc 2017;5(10). pii: e01639-16. 